\title{
X-ray upper limits of GW150914 with MAXI
}

\author{
Nobuyuki Kawal ${ }^{1,2}$, Hitoshi Negoro ${ }^{3}$, Motoko Serino ${ }^{2}$, Tatehiro Mihara ${ }^{2}$, \\ Kazuki TANAKA ${ }^{3}$, Takahiro MASUMITSU ${ }^{3}$ and Satoshi NAKAHIRA ${ }^{2}$ \\ ${ }^{1}$ Department of Physics, Tokyo Institute of Technology, 2-12-1 Ookayama, Meguro-ku, Tokyo \\ $152-8551$ \\ ${ }^{2}$ MAXI team, RIKEN, 2-1 Hirosawa, Wako, Saitama 351-0198, Japan \\ ${ }^{3}$ Department of Physics, Nihon University, 1-8-14 Kanda-Surugadai, Chiyoda-ku, Tokyo \\ 101-8308, Japan
}

Received ; Accepted

\begin{abstract}
We searched for X-ray candidates of the gravitational wave (GW) event GW150914 with Monitor of All-sky X-ray Image (MAXI). MAXI observed the error region of the GW event GW150914 from 4 minutes after the event and covered about $90 \%$ of the error region in 25 minutes. No significant time variations on timescales of $1 \mathrm{~s}$ to 4 days were found in the GW error region. The $3 \sigma$ upper limits for the $\mathrm{X}$-ray emission associated with the $\mathrm{GW}$ event in 2-20 $\mathrm{keV}$ were $9.5 \times 10^{-10}, 2.3 \times 10^{-10}$, and $0.8 \times 10^{-10} \mathrm{ergs}^{-2} \mathrm{~cm}^{-1}$ for the time scale of $\sim 1000$ s, 1 day, and 10 days, respectively. If GW events are associated with short GRBs like GRB 050709, MAXI will be able to detect X-ray emissions from the source.
\end{abstract}

Key words: gravitational waves - methods: observational $-\mathrm{X}$-rays: general

\section{Introduction}

The first detection of the gravitational wave (GW) has been made by LIGO on September 14, 2015, as known as GW150914 (Abbott et al. 2016a). The strain waveform indicates merger of two black holes (BHs) with masses of 29 and $36 M_{\odot}$. It is the first test of the general relativity in the strong field. It is also the first test of the theory for propagation of the gravitational wave in the space. A rough distance of $\sim 400 \mathrm{Mpc}$ was derived by the waveform analysis with a rather large uncertainty. Another important aspect of this event is the first solid evidence for existence of BHs with intermediate masses $\sim 30 M_{\odot}$, and the resulting $\mathrm{BH}$ with $60 M_{\odot}$. Reliable dynamical mass measurements of BHs have been made only for the stellar mass BHs in the Milky Way galaxy and the Large Magellanic Cloud, and the super-massive BHs that lie at centres of galaxies including the Milky Way. The stellar mass BHs whose masses are smaller than a few tens of $M_{\odot}$ are known to be produced by collapse of massive stars at the end of their lives. However, origin of supermassive BHs in nuclei of galaxies is not yet clear. They may be produced by hierarchical mergers of many $\mathrm{BHs}$, or by ac- cretion of gas to the single $\mathrm{BH}$. In either case the massive $\mathrm{BHs}$ at high redshifts indicates that there need to be more massive $\mathrm{BH}$ as the seed. Kinugawa et al. (2014) suggested that BHs of $\sim 30 M_{\odot}$ are naturally produced by collapse of pop-III stars at their endpoints, and that binaries of such BHs are the most common sources of gravitational waves. GW150914 is naturally explained in this scenario, and may imply that the remnants of the Universe's first stars may be found in the neighbourhood of the Milky Way. In order to confirm or test these theories on the birth and evolution of massive $\mathrm{BHs}$, the distance and the environment of the BHs mergers are essential. The poor localization of GW does not allow us to associate the source to any known class of objects such as galaxies, or to know its relative position to its host galaxy.

In order for that we need precise location that is only achievable with electromagnetic (EM) waves. EM counterparts of gravitational wave event have been discussed quite extensively for the case of merger of double neutron star (NS) binary, or NS$\mathrm{BH}$ binaries, where the ejected NS material are supposed to produce EM emission through nuclear decay of r-process elements 
("kilo-nova"; Metzger et al. 2010), accretion on to the newly formed BH (Nakar \& Piran 2011; Piran et al. 2013), or interaction of Blandford-Znajek jet from the rotating BH (Nakamura et al. 2014).

Not so much discussion was made for BH pairs, but some mechanism have been suggested for possible production of EM emission. For example, Nakamura et al. (2016) suggests a mechanism in which the merged $\mathrm{BH}$ could accrete from the interstellar medium to emerge as an EM counterpart.

The possible gamma-ray detection (Connaughton et al. 2016) resembling a weak short gamma-ray burst have prompted theoretical ideas for EM emission. It may not be at all impossible for BH mergers to produce EM emission, if the environment is suitable.

In this paper we present the MAXI follow-up of GW150914. MAXI (Monitor of All-sky X-ray Image; Matsuoka et al. 2009) is an X-ray all-sky monitor on the International Space Station (ISS). It scans most of the sky in every orbit ( 92min) of the ISS with its narrow and long field of view. Most of the error region of GW150914 was covered by MAXI following the event, and placed upper limits on the X-ray emission from the GW event on various time scales.

\section{Instrumentation}

MAXI has two instruments: GSC (2-20 keV; Mihara et al. 2011) and SSC (1-7 keV; Tomida et al. 2011). The instant field of views (FOVs) of GSC and SSC are about $2 \%$ and $1 \%$ of the whole sky. The FOVs scan the whole sky once in 92 minutes. Currently 6 out of 12 GSC cameras are functioning (Mihara et al. 2014). GSC are not operating in the regions with high particle-background, which are South Atlantic Anomaly and higher latitude than $\sim 40$ degrees. The functioning time is about $40 \%$. GSC is turned off in the vicinity of the sun $(\sim 5$ degrees). Still, GSC can cover about $85 \%$ of the whole sky in 92 minutes (Sugizaki et al. 2011). Because SSC is operated in the night time to avoid the sun light, its operating efficiency becomes considerably low. The SSC functioning time and sky coverage in 92 minutes are about $25-30 \%$ and $30 \%$, respectively.

MAXI/GSC is capable to detect transient events with the limit of $\sim 2 \times 10^{-9} \mathrm{erg} \mathrm{cm}^{-2} \mathrm{~s}^{-1}$ in the $2-20 \mathrm{keV}$ band (e.g. Serino et al. 2014; Negoro et al. 2016) in a scan transit.

\section{Observations}

\section{1 time and area of the observation}

MAXI observes a point of the sky every $\sim 92$ minutes. The first GSC observation of the GW150914 region carried out from $t 0$ $(=2015 / 09 / 14$ 09:50:45 UTC) $+4 \min$ to $t 0+25 \min$ (the first scan, hereafter). Figure 1 shows the sky map of the observed

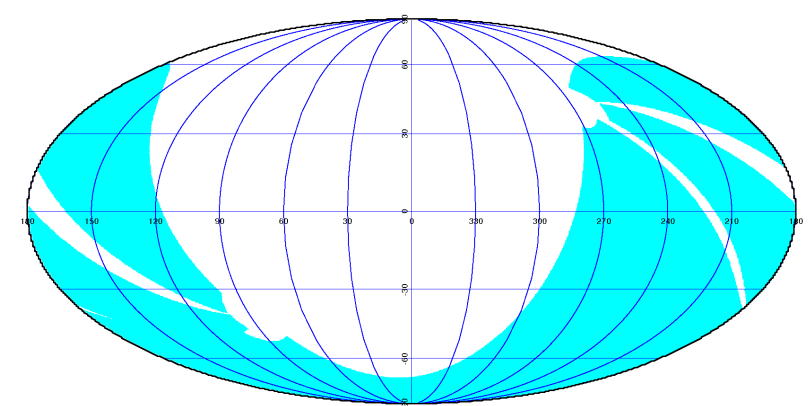

Fig. 1. The schematic picture of the observed region from $t 0+4 \min$ to $t 0$ +25 min (the first scan) by GSC (cyan). The figure is shown in the equatorial coordinates.

area by GSC during the first scan. Figure 2 shows the observed area and scan time of GSC from $t 0+4 \min$ to $t 0+74 \min$ (the first orbit). Probability maps of the GW source position were calculated by various algorithms: Coherent Wave Burst (cWB; Klimenko et al. 2016), LALInference (LALInf; Veitch et al. 2015), LALInference Burst (LIB; Lynch et al. 2015), and BAYESTAR (bay py; Singer \& Price 2016). The region with high significance are observed mainly by GSC_2, GSC_4, and GSC_5.

The SSC observation did not started until $t 0+48$ min, since the ISS entered the day-earth region from $t 0-12 \mathrm{~min}$ to $t 0+44$ min, and also South Atlantic Anomaly from $t 0+35$ min to $t 0+46$ min, Figure 3 shows the all-sky image obtained by two SSC cameras in 1 day.

\section{2 coverage}

We calculated the coverage of the 90 percentile region of each GW skymap by the following procedure. First, we calculated the HEALPix map ${ }^{1}$ of the region which is observed by each GSC camera during the first scan (figure 1). If the center of a pixel is in the field of view of the camera during the time, we regard the pixel as an observed one. Since the pixel size is smaller than the GSC point spread function, it is reasonable. Then we add the maps of all cameras.

Next, we listed the pixel number in the 90 percentile region of each HEALPix map of GW. Then examined in the MAXI map whether the pixels were observed or not. As a result, we obtained the observation coverage within the first scan for each GW map (table 1).

We also show all-sky X-ray images obtained by working 6 GSC cameras from $t 0+4 \min$ to $t 0+74 \min$ (in 1.5 hours; figure 4 ), and in 4 days (figure 5). In producing these GSC images, we did not use GSC_3 data of the region where the GSC_4 had also observed. This was because the background rate in GSC_3 was high due to the loss of the anti-coincident

${ }^{1}$ the pixel size of this GSC map is $\sim 1 \mathrm{deg} \times 1 \mathrm{deg}$ 


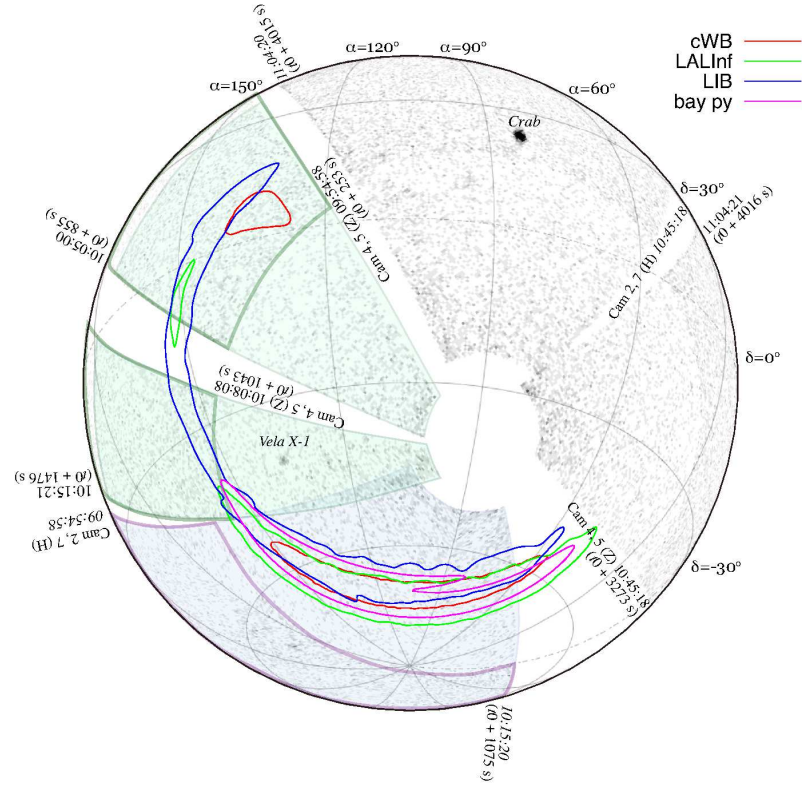

Fig. 2. The schematic picture of the observed area with GSC from $t 0+4$ min to $t 0+74$ min with GW $90 \%$ probability contours by various algorithms (cWB, LALInf, LIB, and bay py). Regions observed with GSC_4 and GSC_7 are surrounded by a green bold-line and a purple one, respectively. Most of the visible parts on this image of the regions are also observed with GSC_5 and GSC_2, respectively.

Table 1. observation coverage of the GW maps in the first scan $(t 0+4 \min$ to $t 0+25$ $\min )$.

\begin{tabular}{llll}
\hline map & pix. 90\%* & pix.obs. ${ }^{\dagger}$ & coverage $^{\ddagger}$ \\
\hline LALInf & 45863 & 38600 & $84.2 \%$ \\
LIB & 56898 & 50451 & $88.7 \%$ \\
cWB & 1469 & 1395 & $95.0 \%$ \\
bay py & 33440 & 30702 & $91.8 \%$ \\
\hline * number of the HEALPix pixels contained in the 90 \\
percentile region of each map \\
${ }^{\dagger}$ number of the pixels contained in the 90 percentile region \\
of each map and observed by MAXI \\
${ }^{\ddagger}$ observation coverage of 90 percentile region by MAXI
\end{tabular}

background rejection function (Mihara et al. 2014).

We also did not use the events detected with the anode 5 of the GSC_5 because solar soft X-rays made a fake point-like image around the region $(\alpha, \delta)=(159,16)$. As can be seen from these images, GSC observed about $80 \%$ and $95 \%$ of the whole sky in 1.5 hours and in 1 day, respectively. SSC covered $39 \%$ of the whole sky in 1 day (figure 3).

\subsection{Event search by the nova-alert system}

The MAXI nova-alert system (Negoro et al. 2016) detected no significant time variability in any error regions for 4 days since the GW trigger time. The nova-alert system consists of a novasearch system(s) to find time variability and an alert system to eliminate further statistical significance of the events triggering the nova-search system.

In figure 6, we plot locations of triggered events in one of two nova-search systems (a system with relatively high event thresholds) for the first 4 days around the error regions. The diamonds represent short-term events that triggered in 1.5 hours in $1 \mathrm{~s}, 3 \mathrm{~s}, 10 \mathrm{~s}, 30 \mathrm{~s}$, and 1 orbit ( $\simeq 92 \mathrm{~min})$ integrated-time bins. The squares show long-term events triggered from $t 0+1$ orbit to $t 0+4$ orbits (in 4 orbits bin), from $t 0+4$ orbits to $t 0+1$ day ( $1 \mathrm{~d}$ bin) and from $t 0+1$ day to $t 0+4$ days ( $4 \mathrm{~d}$ bin). The colors, black, red, green, and blue, of the marks represent energy bands triggered, corresponding to the $3-10 \mathrm{keV}, 2-4 \mathrm{keV}, 4-10 \mathrm{keV}$, and $10-20 \mathrm{keV}$ energy band, respectively. Different mark sizes for different energy band data are to avoid overwriting, and does not show any significance. Chance probabilities to trigger, i.e., the trigger criteria, are $\leq 10^{-3}$ to $10^{-4}$.

The circles show detected events, related with the triggered events for 4 days, which meet detected criteria as statistically significant events in the alert system (Negoro et al. 2016). As described previously, no event was detected in any of the $90 \%$ probability regions. Bright catalogued sources, such as Cen X-3 and Vela $\mathrm{X}-1$, and their neighborhoods often triggered the system, but are masked in the alert system.

Triggered events without circles are not statistically significant (usually at less than $3 \sigma$ levels), but are candidates of variable events. An event $\mathbf{A}$ at $(\alpha, \delta)=(132.43,6.73)$ in the cWB error regions is noticeable because it first triggered at 09:57:42 $(t 0+417 \mathrm{~s})$ in 30 -s and 1 -orbit time bins. The $4-10 \mathrm{keV}$ flux at the region in the scan transit at 09:57 was $0.035_{-0.015}^{+0.018}$ counts $\mathrm{cm}^{-2} \mathrm{~s}^{-1}$, and we could not confirm any point-source like excess for this event in GSC images.

An event B at $(\alpha, \delta)=(150.44,-10.55)$ near the LALInf $90 \%$ probability region and in the LIB one triggered the system from 11:37:36 $(\sim t 0+107 \mathrm{~min})$ from 13:10:19 $(\sim t 0+200 \mathrm{~min})$ in the 4 orbits bin. These events, however, are due to the noise caused by the reduction of high voltage of the counters and not astronomical events.

\section{4 upper limits of the flux}

We evaluated the upper limits of the flux by the following procedure. First, we selected 10 points representing the observed region and counted the photons in the circular regions with the radii of $1.5 \mathrm{deg}$, which is the typical size of the PSF. The 1sigma fluctuation of the background is defined as $\sqrt{n}$, where $n$ is observed count in the circular region. Next, we calculated the effective exposure $a$, which has the dimension of area $\times$ time, of each of the 10 points. Then we regarded $f \equiv 3 \sqrt{n} / a$ as 3-sigma upper limit of the flux at the point. The averages of 3-sigma upper limits of the points for the observations of a scan is $0.12 \pm 0.02 \mathrm{c} \mathrm{s}^{-1} \mathrm{~cm}^{-2}$ (in 2-20 keV), which corresponds to the energy flux of $(9.5 \pm 1.8) \times 10^{-10} \mathrm{erg} \mathrm{s}^{-1} \mathrm{~cm}^{-2}$. 


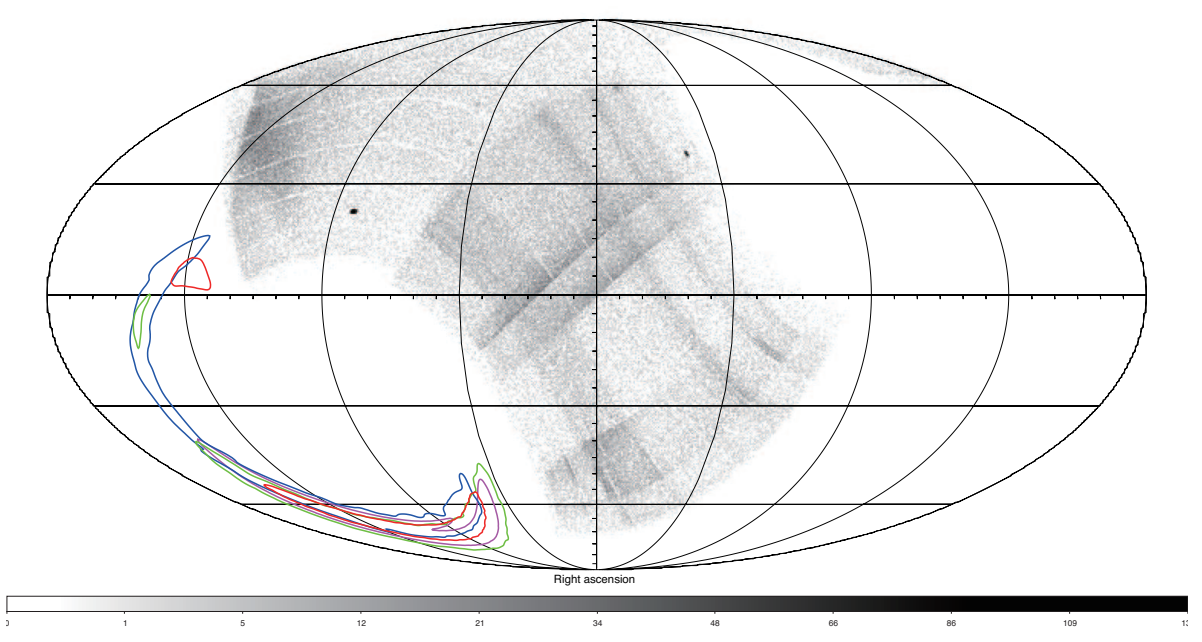

Fig. 3. A single pixel event X-ray image observed by SSC from $t 0+48$ min to $t 0+1$ day. The GW $90 \%$ probability contours with the same colors in figure 2 were also shown.

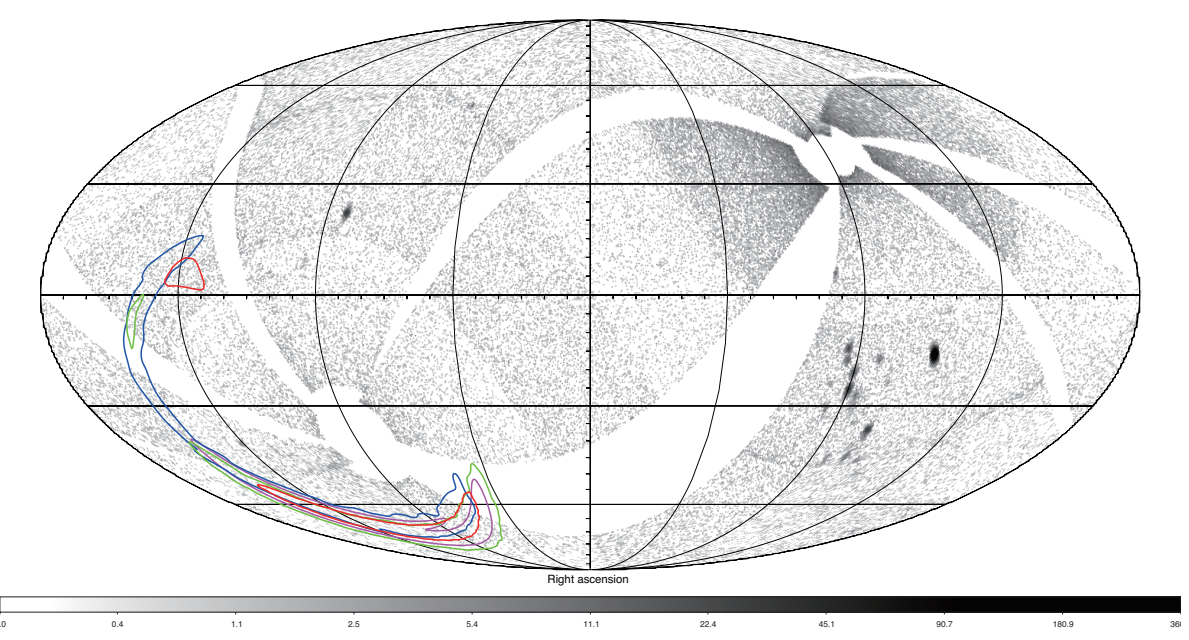

Fig. 4. An X-ray image observed by GSC from $t 0+4 \min$ to $t 0+74 \mathrm{~min}$. GW contours are same as figure 3 .

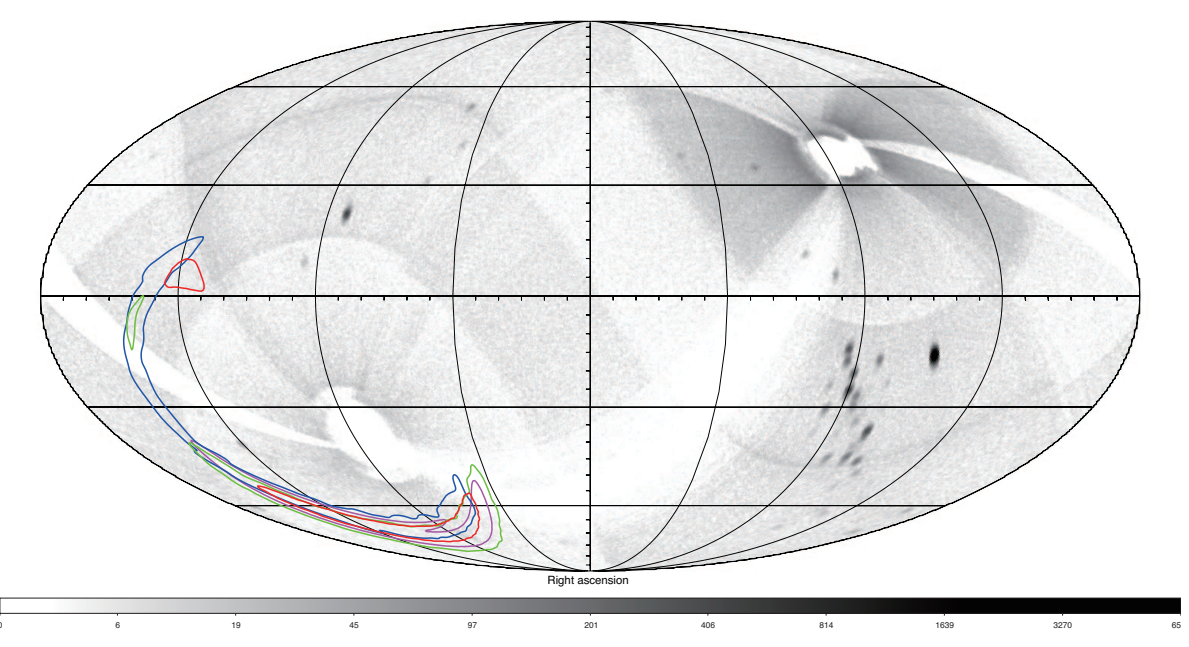

Fig. 5. An X-ray image observed by GSC from $t 0+4$ min to $t 0+1$ day. GW contours are same as figure 3 . 


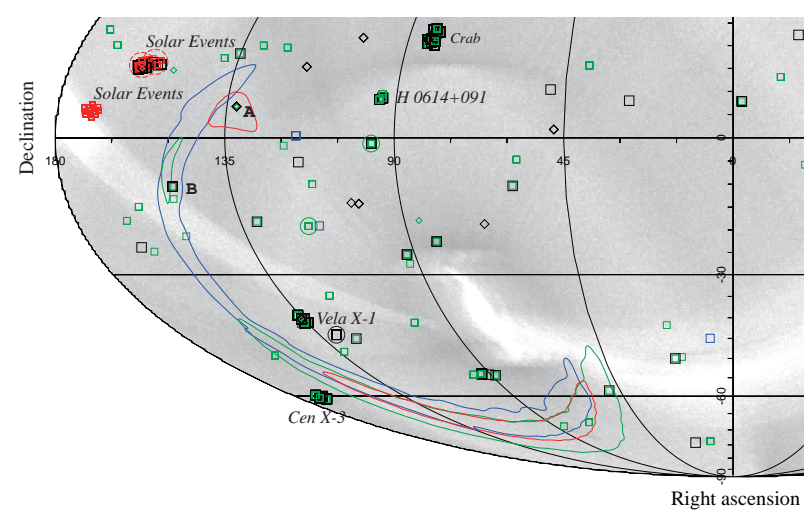

Fig. 6. Locations of the triggered events in a nova-search system in the first orbit (shown by triangles) and in 4 days (squares), and those of more significantly detected events (circles, see text for more detail), overwriting on the 4-day GSC 2-20 keV image. Only the $90 \%$ probability contours are shown, and the bay-py contour is omitted to avoid complexity. Events shown in red (and black) in the upper left side are due to solar X-rays.

The upper limits for the one day and ten days observations in the same energy band are of a scan $0.029 \pm 0.004 \mathrm{c} \mathrm{s}^{-1} \mathrm{~cm}^{-2}$ $\left[(2.3 \pm 0.4) \times 10^{-10} \mathrm{erg} \mathrm{s}^{-1} \mathrm{~cm}^{-2}\right]$ and of a scan $0.010 \pm 0.001$ $\mathrm{c} \mathrm{s}^{-1} \mathrm{~cm}^{-2}\left[(8.2 \pm 1.0) \times 10^{-11} \mathrm{erg} \mathrm{s}^{-1} \mathrm{~cm}^{-2}\right]$, respectively.

\section{4 discussion}

The upper limits on the X-ray flux on three different time scales can be summarized in table 2. The upper limits for the energy radiated in X-ray over the measurement time scale are also shown, and compared with the energy radiated in gravitational wave. These can be treated as upper limits for the extended X-ray afterglow of the GW event. For example, we estimate that the energy radiated in X-ray afterglow over 1000 seconds is less than $3.5 \times 10^{-6}$ of the total energy released in the $\mathrm{BH}$ merger. Since GW150914 is a BH binary merger, strong X-ray emission is not naturally expected. In that respect, our upper limits do not constrain the theory or information on the environment. On the other hand, possible detection of a weak short gamma-ray transient similar to a short GRB by Fermi GBM was reported (Connaughton et al. 2016). The future observation of LIGO with improved sensitivity is expected to detect gravitational waves from mergers of double NSs or BH-NS binaries are expected. For these systems, significant fraction of NS matter is expected to be ejected. Theories predicts various ways of generating electromagnetic radiation from these events resulting from the radiative decays of r-process nuclei (kilonova) and free neutrons, or the relativistic jet powered by the central engine such as accreting $\mathrm{BH}$ or rotating magnetic compact objects through the Blandford-Znajek process. In particular, the latter is considered as the promising origin of short gamma-ray bursts, that emits intense gamma-ray radiation followed by extended $\mathrm{X}$-ray emission.

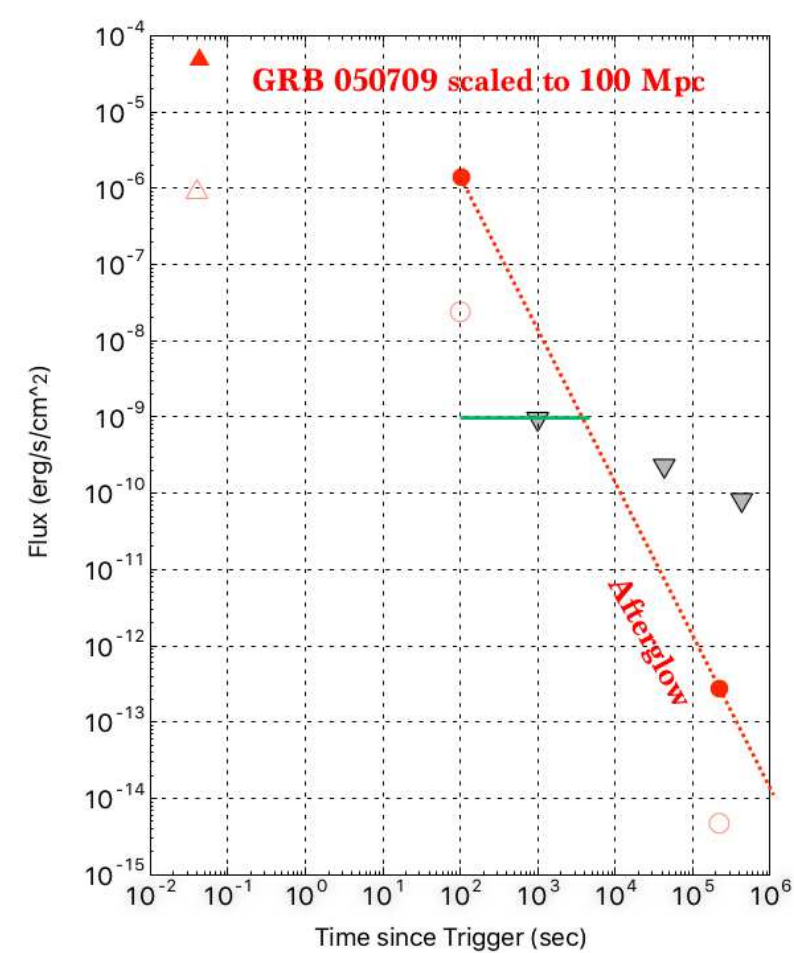

Fig. 7. The upper limits for the $2-20 \mathrm{keV} X$-ray flux associated with GW150914 on three time scales, 1000s, 1 day, and 10 days, are shown with gray down-pointing triangles. They are compared with the X-ray fluxes of GRB050709 at three different phases: short pulse, extended emission, and afterglow shown by open triangle and circles. These fluxes are then scaled to the source distance of $100 \mathrm{Mpc}$ and plotted with filled symbols. The dashed line indicate a possible afterglow light curve connecting the soft $\mathrm{X}$-ray extended emission and the late afterglow.

It is interesting to compare the MAXI sensitivity to the X-ray flux expected from the possible gamma-ray transient recorded by Fermi GBM. Using the photon power-law index -1.4 and fluence between 10 and $1000 \mathrm{keV}$ of $2.4 \times 10^{-7} \mathrm{erg} \mathrm{cm}^{-2}$ reported for this event, we estimate the $2-20 \mathrm{keV}$ fluence of $\sim 2 \times 10^{-8} \mathrm{erg} \mathrm{cm}^{-2}$. If the event were captured at the middle of the scan transit, it would produce a significant detection with $\sim 10$ counts in a MAXI GSC camera in less than a second, which is more than an order of magnitude higher than the background of GSC (Sugizaki et al. 2011) for that duration. In general, however, MAXI has only a small chance for detecting prompt emission coincident with the gravitational wave, because the instantaneous sky coverage of MAXI is only $2 \%$ of the entire sky. We need to wait for more GW events to be detected for such a luck.

It is also instructive to see how the present MAXI upper limits for GW150914 are compared with a possible future detection of a short GRB coincident with GW detection. In Fig. 7 we plot the MAXI upper limits for the X-ray flux as a function of the time since the GW150914 trigger. The three points are naturally aligned on a straight line on a logarithmic plot following a $\propto t^{-1 / 2}$ relation expected for the background-limited sensitiv- 
Table 2. Upper limits for the X-ray flux and radiated energy obtained by MAXI/GSC.

\begin{tabular}{ccclll}
\hline & $\begin{array}{c}\text { Timescale } \\
(\mathrm{s})\end{array}$ & $\begin{array}{l}\text { Flux }(2-20 \mathrm{keV}) \\
\left(\mathrm{erg} \mathrm{s}^{-1} \mathrm{~cm}^{-2}\right)\end{array}$ & $\begin{array}{l}\text { Luminosity } \\
\left(\mathrm{erg} \mathrm{s}^{-1}\right)\end{array}$ & $\begin{array}{l}\text { Radiated } \\
\text { Energy }(\mathrm{erg})\end{array}$ & $E_{X} / E_{G W}$ \\
\hline 1 orbit & 1000 & $<9.5 \times 10^{-10}$ & $<1.9 \times 10^{46}$ & $<1.9 \times 10^{49}$ & $<3.5 \times 10^{-6}$ \\
1 day & $8.6 \times 10^{4}$ & $<2.3 \times 10^{-10}$ & $<4.6 \times 10^{45}$ & $<4.0 \times 10^{50}$ & $<7.4 \times 10^{-5}$ \\
10 days & $8.6 \times 10^{5}$ & $<0.8 \times 10^{-10}$ & $<1.6 \times 10^{45}$ & $<1.4 \times 10^{51}$ & $<2.6 \times 10^{-4}$ \\
\hline
\end{tabular}

* Distance of $410 \mathrm{Mpc}$ assumed

ity.

We can also compare the MAXI sensitivity with the X-ray flux of GRB 050709, a short GRB. We choose this short GRB for comparison, since it is the only short GRB for which the prompt burst phase has been observed in the energy band common with MAXI/GSC. No other GRB missions like Swift and Fermi have comparable sensitivity in the X-ray band below 10 $\mathrm{keV}$. The WXM on HETE-2 observed the prompt short-hard pulse with duration $\sim 0.3 \mathrm{~s}$ and the extended soft $\mathrm{X}$-ray emission that lasted $>100 \mathrm{~s}$ in the $2-25 \mathrm{keV}$ X-ray band (Villasenor et al. 2005). Its X-ray afterglow was detected by Chandra, which lead to Hubble detection of optical afterglow and identification of the host galaxy at $z \approx 0.16$ with the precise localization (Fox et al. 2005). We plot its X-ray fluxes in the short hard pulse, the extended X-ray emission, and the afterglow with open symbols in Fig. 7. These fluxes are scaled to the source distance of $100 \mathrm{Mpc}$, the expected range for double NS merger with LIGO O2 (Abbott et al. 2016b). It is immediately clear that the prompt X-ray emission, both short pulse and extended emission, of GRB 050709 is far brighter than the detection threshold of MAXI/GSC even at its original redshift, not to mention the case scaled to the LIGO $\mathrm{O} 2$ range. Here after we discuss the possibility for detecting the X-ray emission if a short GRB is associated with the NS merger event in the LIGO O2 run. Despite this high flux, the chance for detecting short pulse is expected to be very low because of the narrow collimation of short GRBs emission (Fong et al. 2015) and MAXI's small instantaneous sky coverage. While there is strong evidence for the short pulse originating in a relativistic jet with small opening angle, the nature and origin of the soft extended emission remains a mystery. If the collimation of the soft X-ray extended emission is weak, as in the model proposed by Nakamura et al. (2014), the chance for MAXI detection may not be negligible. The MAXI sky coverage may be still a problem, since the duration of soft extended emission is much shorter than the scan interval of MAXI of 92 minutes, the ISS orbital period. However, if the soft extended emission is connected to the late afterglow as indicated by a dashed line in Fig. 7, its flux stays above the MAXI threshold for more than $3000 \mathrm{~s}$, a major fraction of the scan interval, suggesting a higher probability for detection.

In summary, MAXI set an upper limit for the X-ray emission associated with the gravitational wave event GW150914 on the timescales of one orbit ( $\sim 1000 \mathrm{~s})$, day, and 10 days following the $\mathrm{GW}$ trigger. In the future $\mathrm{GW}$ observing runs, MAXI has possibility to constrain the model for electromagnetic radiation and association of GW events with short GRBs.

\section{Acknowledgments}

This research has made use of the MAXI data provided by RIKEN, JAXA and the MAXI team. This research was supported by JSPS KAKENHI Grant Number 24740186.

\section{References}

Abbott, B. P., Abbott, R., Abbott, T. D., et al. 2016a, Physical Review Letters, 116, 061102

Abbott, B. P., Abbott, R., Abbott, T. D., et al. 2016b, Living Reviews in Relativity, 19

Connaughton, V., Burns, E., Goldstein, A., et al. 2016, ApJL, 826, L6

Fong, W., Berger, E., Margutti, R., \& Zauderer, B. A. 2015, ApJ, 815 , 102

Fox, D. B., Frail, D. A., Price, P. A., et al. 2005, Nature, 437, 845

Kinugawa, T., Inayoshi, K., Hotokezaka, K., Nakauchi, D., \& Nakamura, T. 2014, MNRAS, 442, 2963

Klimenko, S., Vedovato, G., Drago, M., et al. 2016, Phys. Rev. D, 93 , 042004

Lynch, R., Vitale, S., Essick, R., Katsavounidis, E., \& Robinet, F. 2015, ArXiv e-prints

Matsuoka, M., Kawasaki, K., Ueno, S., et al. 2009, PASJ, 61, 999

Metzger, B. D., Martínez-Pinedo, G., Darbha, S., et al. 2010, MNRAS 406, 2650

Mihara, T., Nakajima, M., Sugizaki, M., et al. 2011, PASJ, 63, 623

Mihara, T., Sugizaki, M., Matsuoka, M., et al. 2014, in Proc. SPIE, Vol. 9144, Space Telescopes and Instrumentation 2014: Ultraviolet to Gamma Ray, 914410

Nakamura, T., Kashiyama, K., Nakauchi, D., et al. 2014, ApJ, 796, 13

Nakamura, T., Nakano, H., \& Tanaka, T. 2016, Phys. Rev. D, 93, 044048

Nakar, E. \& Piran, T. 2011, Nature, 478, 82

Negoro, H., Kohama, M., Serino, M., et al. 2016, PASJ, 68, S1

Piran, T., Nakar, E., \& Rosswog, S. 2013, MNRAS, 430, 2121

Serino, M., Sakamoto, T., Kawai, N., et al. 2014, PASJ, 66, 87

Singer, L. P. \& Price, L. R. 2016, Phys. Rev. D, 93, 024013

Sugizaki, M., Mihara, T., Serino, M., et al. 2011, PASJ, 63, 635

Tomida, H., Tsunemi, H., Kimura, M., et al. 2011, PASJ, 63, 397

Veitch, J., Raymond, V., Farr, B., et al. 2015, Phys. Rev. D, 91, 042003

Villasenor, J. S., Lamb, D. Q., Ricker, G. R., et al. 2005, Nature, 437, 855 